\title{
Anti-varicella-zoster virus activity of cephalotaxine esters in vitro
}

\author{
Jung-Eun Kim and Yoon-Jae Song* \\ Department of Life Science, Gachon University, Seongnam 13120, \\ Republic of Korea \\ (Received Sep 17, 2018 / Revised Oct 2, 2018 / Accepted Oct 4, 2018)
}

Harringtonine (HT) and homoharringtonine (HHT), alkaloid esters isolated from the genus Cephalotaxus, exhibit antitumor activity. A semisynthetic HHT has been approved for treatment of chronic myelogenous leukemia. In addition to antileukemic activity, HT and HHT are reported to possess potent antiviral activity. In this study, we investigated the effects of HT and HHT on replication of varicella-zoster virus (VZV) in vitro. HT and HHT, but not their biologically inactive parental alkaloid cephalotaxine (CET), significantly inhibited replication of recombinant VZV-pOka luciferase. Furthermore, HT and HHT, but not CET, strongly induced down-regulation of VZV lytic genes and exerted potent antiviral effects against a VZV clinical isolate. The collective data support the utility of HT and HHT as effective antiviral candidates for treatment of $\mathrm{VZV}$-associated diseases.

Keywords: varicella zoster virus, antiviral, harringtonine, homoharringtonine

\section{Introduction}

Varicella-zoster virus (VZV), a member of the herpesvirus family, is a causative agent of varicella (chickenpox) and zoster (shingles) (Arvin, 1996; Arvin and Gilden, 2013). VZV is highly species-specific with its natural host range restricted to humans. Primary VZV infection in childhood causes a diffuse vesicular rash of varicella, followed by resolution of illness by the host immune system and establishment of latent VZV infection in the dorsal root ganglia. Reactivation of latent VZV infection results in herpes zoster, which provokes severe pain associated with postherpetic neuralgia (Arvin, 1996; Arvin and Gilden, 2013).

Inhibitors of VZV DNA polymerase are currently employed in the clinic to treat VZV-associated diseases (Cohen et al., 1999). Acyclovir and famciclovir/penciclovir are nucleoside analogs which lacks the $3^{\prime}-\mathrm{OH}$ for the phosphodiester bond between nucleotides. Upon activation by viral thymidine kinase (TK), these drugs are incorporated into the viral DNA synthesis to prevent DNA elongation (Cohen et al., 1999). Foscarnet, a pyrophosphate analog, binds the pyro-

${ }^{\star}$ For correspondence. E-mail: songyj@gachon.ac.kr; Tel.: +82-31-750-8731; Fax: +82-31-750-4797

Copyright (C) 2019, The Microbiological Society of Korea phosphate binding site to inhibit viral DNA polymerase (Cohen et al., 1999; Gnann Jr, 2007). Although these antiVZV dugs are highly effective, more concerted efforts to develop novel therapeutic strategies for VZV-associated diseases are necessary due to the emergence of drug resistance and side-effects of available antiviral agents (Ida et al., 1999; Arvin, 2002).

Members of the genus Cephalotaxus produce various alkaloids, such as cephalotaxine (CET), harringtonine (HT), homoharringtonine (HHT), isoharringtonine, and deoxyharringtonine (Lu and Wang, 2014). CET, a benzazepinebearing pentacyclic alkaloid, is a parent structure of $\mathrm{C} 3$-ester compounds, including HT and HHT (Fig. 1). HT is a C3 ahydroxyl succinate ester of CET while HHT contains a methylene group in the side-chain (Quintas-Cardama et al.,

(A) HHT<smiles>COC(=O)CC(O)(CCCC(C)(C)O)C(=O)OC1C(OC)=CC23CCCN2CCc2cc4c(cc2C13)OCO4</smiles>

(B) HT<smiles>COC(=O)CC(O)(CCC(C)(C)O)C(=O)OC1C2C=C(OC)C34CCCN3CCc3cc5c(cc3C1C24)OCO5</smiles>

(C) CET<smiles>CC1=CC23CCCN2CCc2cc4c(cc2C3C1O)OCO4</smiles>

Fig. 1. Structures of Cephalotaxus alkaloids used in this study. (A) HHT, (B) HT, (C) CET. 
2009). Although CET is biologically inactive, HT and HHT exert potent antileukemic effects (Powell et al., 1972). Indeed, omacetaxine mepesuccinate, a semisynthetic HHT produced by direct esterification of CET, has been approved by the Food and Drug Administration (FDA) for treatment of chronic myelogenous leukemia (CML) patients resistant or intolerant to tyrosine kinase inhibitors (Tujebajeva et al., 1992; Chen et al., 2011).

In addition to antileukemic effects, HT and HHT are reported to exhibit antiviral activity. HT inhibits chikungunya virus (CHIKV) replication through down-regulation of viral protein expression while HHT displays activity against hepatitis B virus (HBV) and coronavirus (Romero et al., 2007; Kaur et al., 2013; Cao et al., 2015). In the current study, we investigated whether HT and HHT exert antiviral effects against $\mathrm{VZV}$, in accordance with previous reports.

\section{Materials and Methods}

\section{Cells, viruses, chemicals, and biological assays}

Primary human foreskin fibroblast (HFF) cells were cultured in Dulbecco's modified eagle's medium (GE Healthcare Life Sciences) supplemented with $10 \%$ fetal bovine serum (Capricorn Scientific), penicillin $(100 \mathrm{U} / \mathrm{ml})$ and streptomycin $(100 \mu \mathrm{g} / \mathrm{ml})$. Recombinant VZV-pOka expressing luciferase reporter (VZV-pOka-luciferase) and the clinical YC01 strain of VZV (VZV-YC01) have been described previously (Zhang et al., 2007; Jeon et al., 2016). HT and HHT were purchased from Santa Cruz Biotechnology and Sigma-Aldrich, respectively. CET was obtained from Glentham Life Sciences and acyclovir (ACV) from Calbiochem. The luciferase activities of VZV-pOka-luciferase were measured using the luciferase assay system from Promega. The CellTiter-Glo luminescent cell viability assay was performed according to the manufacturer's instructions (Promega). Plaque reduction assay was performed as described previously (Bae et al., 2017).

\section{Quantification of VZV DNA and transcripts}

For quantitative analysis of VZV DNA, quantitative polymerase chain reaction ( $\mathrm{PPCR}$ ) was employed as described previously (Bae et al., 2017). Briefly, total DNA was isolated using an AccuPrep Genomic DNA Extraction kit (Bioneer), and VZV DNA was amplified and quantified on a StepOnePlus Real-Time PCR system (Applied Biosystems) using HOT FIREPol ${ }^{\circledR}$ EvaGreen qPCR master mix Plus (Solis BioDyne) and specific primers for amplification of VZV or human GAPDH DNA. VZV transcripts were analyzed via quantitative reverse transcription PCR (qRT-PCR) according to a previous report (Bae and Song, 2017). The primer sequences used for amplification were as follows: VZV ORF63 (IE), forward 5' -ATTGAGGCGCCGAATGTTC-3' and reverse 5'-CTTCACCACCATCAGATACG-3'; VZV ORF28 (E), forward $5^{\prime}$ - CGAACACGTTCCCCATCAA- $3^{\prime}$ and reverse $5^{\prime}$ CCCGGCTTTGTTAGTTTTGG-3'; VZV gB, forward 5'-GA TGGTGCATACAGAGAACATTCC- $3^{\prime}$ and reverse $5^{\prime}$-CCG TTAAATGAGGCGTGACTAA-3'; GAPDH, forward $5^{\prime}$-C ATGAGAAGTATGACAACAGCCT- $3^{\prime}$ and reverse $5^{\prime}$-AGT CCTTCCACGATACCAAAGT-3'.

\section{Western blot analysis}

Cells were harvested, fractionated and transferred onto nitrocellulose membrane, as described previously (Kim et al., 2014). Antibodies against VZV ORF62 and tubulin were purchased from Abcam and Sigma-Aldrich, respectively. Secondary peroxidase-labeled anti-mouse immunoglobulin $G$ antibodies (Amersham Biosciences) and enhanced chemiluminescence detection reagent for visualization of proteins (Pierce) were used according to the manufacturer's specifications.

\section{Results}

\section{Anti-VZV activities of ester alkaloids from Cephalotaxus}

To investigate the potential anti-VZV activities of alkaloids from Cephalotaxus, uninfected HFF cells were inoculated

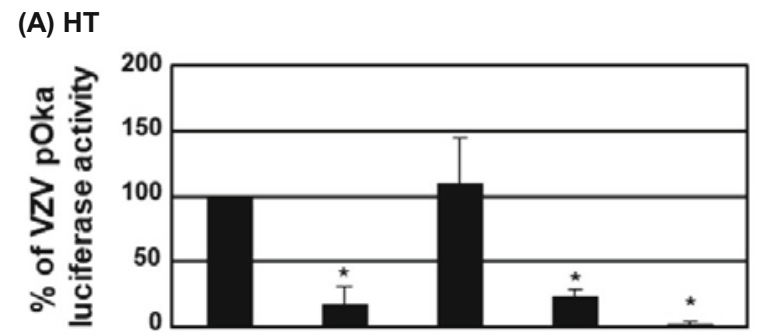

(B) $\mathrm{HHT}$

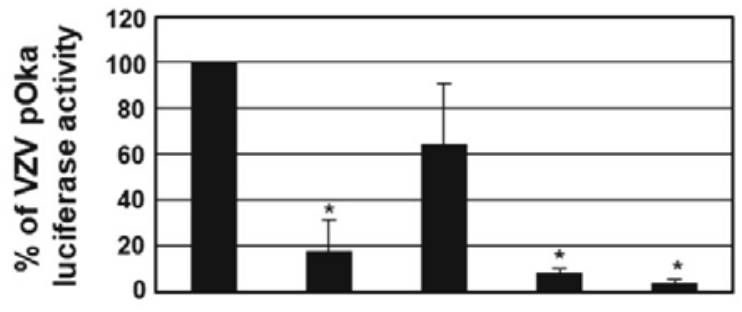

(C) CET

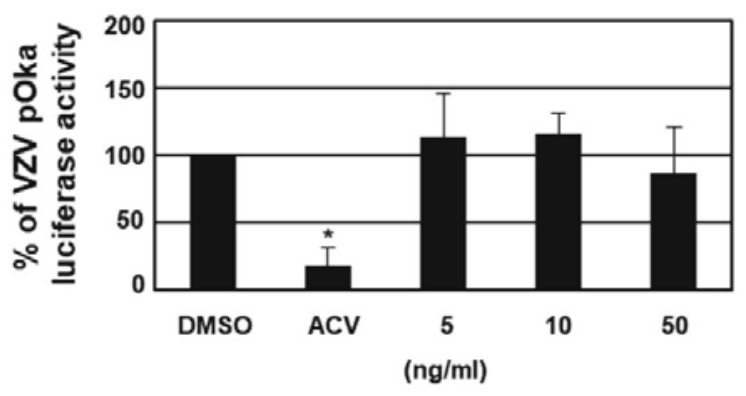

Fig. 2. Inhibition of VZV-pOka-luciferase activity by HT and HHT. HFF cells were inoculated with VZV-pOka-luciferase-infected HFF cells at an MOI of 0.1 and treated with (A) HT, (B) HHT, or (C) CET at concentrations of 5,10 , and $50 \mathrm{ng} / \mathrm{ml}$. Cells treated with ACV $(5 \mu \mathrm{M})$ served as a positive control. Luciferase activities were measured at $72 \mathrm{~h}$ after inoculation. VZV pOka-luciferase activity in DMSO-treated cells was set as $100 \%$. Data are represented as means \pm SD of three independent experiments. The asterisk $\left({ }^{*}\right)$ denotes a significant difference between samples determined using the two-sample $t$-test $(P<0.05)$. 
with VZV-pOka-luciferase-infected HFF cells at an MOI of 0.1 , followed by treatment with DMSO, acyclovir, HT, HHT or CET. At $72 \mathrm{~h}$ after inoculation, VZV replication was determined based on luciferase activity (Fig. 2). Both HT and HHT reduced VZV-pOka-luciferase activity in a dose-dependent manner (Fig. 2A and B), while CET had no effect (Fig. 2C). Acyclovir treatment at a concentration of $5 \mu \mathrm{M}$ $(\sim 1.13 \mu \mathrm{g} / \mathrm{ml})$ reduced VZV-pOka-luciferase activity by $82 \%$. Interestingly, both HT and HHT were more potent than acyclovir reducing VZV-pOka-luciferase activity by $76.1 \%$ and $91.3 \%$, respectively, at a concentration of $10 \mathrm{ng} / \mathrm{ml}$. Overall, HHT was more effective than HT in inhibiting replication of VZV-pOka-luciferase, with $50 \%$ effective concentration $\left(\mathrm{EC}_{50}\right)$ values (at which VZV replication was reduced by $50 \%$ ) of $4.654 \pm 1.134 \mathrm{ng} / \mathrm{ml}$ and $9.574 \pm 3.474$ $\mathrm{ng} / \mathrm{ml}$ for HHT and HT, respectively.

To determine whether the anti-VZV activities of Cephalotaxus ester alkaloids are mediated via toxic effects on cells,

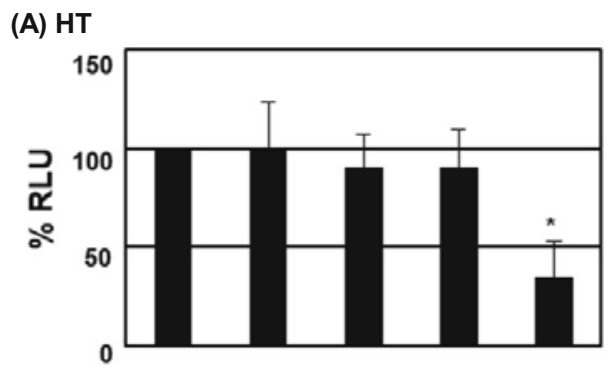

(B) HHT

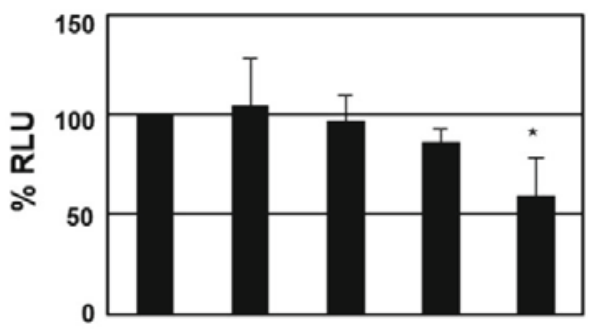

(C) CET

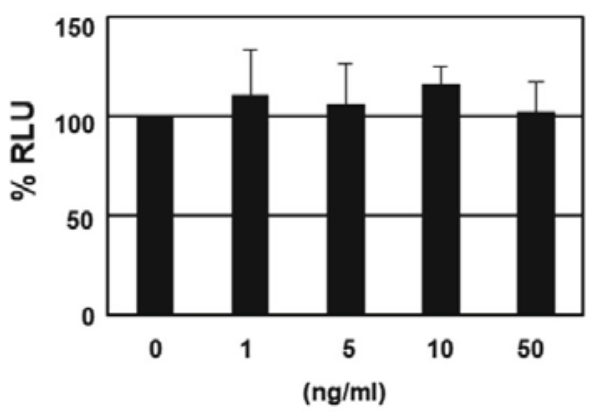

Fig. 3. Cytotoxic effects of HT, HHT and CET against HFF cells. HFF cells were treated with (A) HT, (B) HHT, or (C) CET at concentrations of 1, 5, 10 , and $50 \mathrm{ng} / \mathrm{ml}$. At $72 \mathrm{~h}$ after treatment, cell viability was determined using the CellTiter-Glo luminescent cell viability assay. Luciferase activity in DMSO-treated cells $(0 \mathrm{ng} / \mathrm{ml})$ was set as $100 \%$. Data are represented as means \pm SD of three independent experiments. The asterisk $\left(^{*}\right)$ denotes a significant difference between samples determined using the two-sample $t$-test $(P<0.05)$.
HFF cells were treated with $0,1,5,10$, or $50 \mathrm{ng} / \mathrm{ml} \mathrm{HT}$, HHT and CET, and cell viability assessed by determining cellular ATP levels using the CellTiter-Glo assay after $72 \mathrm{~h}$ (Fig. 3). HT, HHT, and CET exhibited no significant cytotoxicity up to $10 \mathrm{ng} / \mathrm{ml}$ against HFF cells (Fig. 3). At a dose of $50 \mathrm{ng} / \mathrm{ml}$, HT and HHT suppressed HFF cell viability by $65.3 \%$ and $40.4 \%$, respectively (Fig. 3A and B). The 50\% cytotoxicity concentration $\left(\mathrm{CC}_{50}\right)$ values of HT and HHT for HFF cells

\section{(A) ORF63}

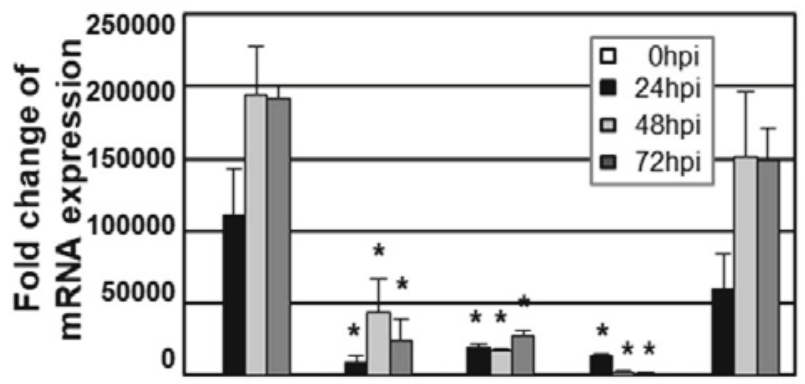

(B) ORF28

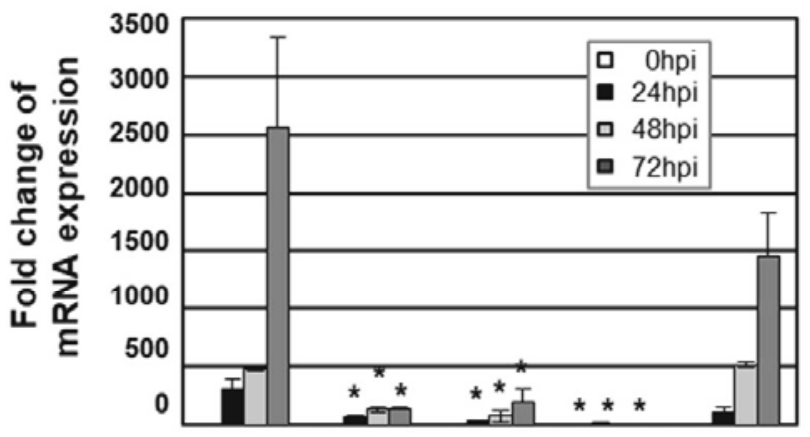

(C) $g B$

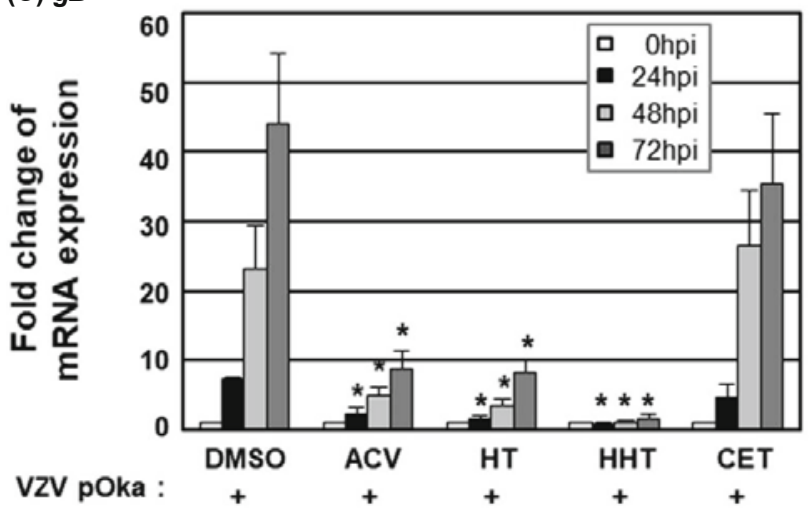

Fig. 4. Down-regulation of VZV lytic gene transcripts by HT and HHT. HFF cells were inoculated with VZV-pOka-luciferase-infected HFF cells at an MOI of 0.1 and treated with DMSO, HT, HHT or CET $(10 \mathrm{ng} / \mathrm{ml})$. HFF cells treated with ACV $(5 \mu \mathrm{M})$ served as a positive control. At 24, 48, or $72 \mathrm{~h}$ post inoculation (hpi), total RNA was harvested and reversetranscribed into cDNA. Relative amounts of VZV [ORF63 (IE), ORF28 (E), or gB (L)] transcripts were measured via qRT-PCR, as described in 'Materials and Methods'. The transcript levels at 0 hpi were set as 1 . Data are represented as means $\pm \mathrm{SD}$ of three independent experiments. The asterisk $\left({ }^{*}\right)$ denotes a significant difference between samples determined using the two-sample $t$-test $(P<0.05)$. 


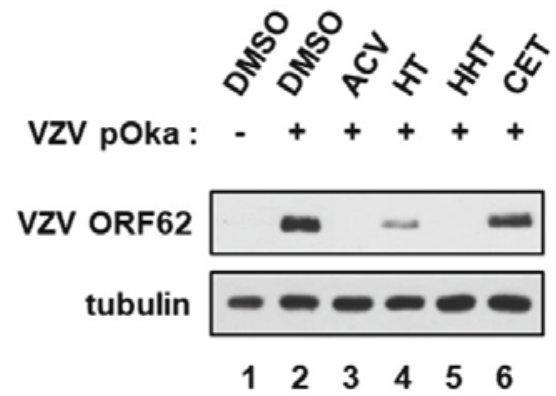

Fig. 5. Down-regulation of VZV ORF62 protein by HT and HHT. HFF cells were inoculated with VZV-pOka-infected HFF cells at an MOI of 0.1 and treated with DMSO, HT, HHT, or CET $(10 \mathrm{ng} / \mathrm{ml})$. HFF cells were also treated with ACV $(5 \mu \mathrm{M})$ served as a positive control. At $72 \mathrm{~h}$ after infection, equal amounts of cell extracts were subjected to western blot with antibodies against VZV ORF62 or tubulin.

(at which cell viability was reduced by $50 \%$ ) were determined as $45.82 \pm 12.72 \mathrm{ng} / \mathrm{ml}$ and $74.71 \pm 15.76 \mathrm{ng} / \mathrm{ml}$, respectively. In view of the finding that the anti-VZV activities of HT and $\mathrm{HHT}$ at $10 \mathrm{ng} / \mathrm{ml}$ do not appear to be mediated through cytotoxic effects, this dose was used for subsequent studies.

\section{Effects of ester alkaloids from Cephalotaxus on VZV lytic gene expression}

During the lytic cycle of infection, VZV lytic genes are expressed in a sequential cascade composed of immediate early (IE), early (E), and late (L) genes (Arvin and Gilden, 2013). To determine the effects of HT and HHT on VZV lytic gene expression patterns, uninfected HFF cells were inoculated with VZV-pOka-luciferase-infected HFF cells at an MOI of 0.1 , followed by treatment with DMSO, acyclovir, HT, HHT, or CET. At 24, 48, and $72 \mathrm{~h}$ after inoculation, the relative transcript levels of ORF63 encoding IE63 (IE), ORF28 encoding a subunit of viral DNA polymerase (E) and glycoprotein $\mathrm{B}(\mathrm{gB})(\mathrm{L})$ were analyzed via qRT-PCR (Fig. 4). HT treatment reduced ORF63, ORF28, and gB transcript levels by $85.5 \%, 92.6 \%$, and $81.2 \%$ while HHT suppressed the levels by $99.2,99.8$, and $96.4 \%$ at $72 \mathrm{~h}$ after inoculation (Fig. 4). On the other hand, CET exerted no effects on VZV lytic gene expression (Fig. 4). Furthermore, HT and HHT, but not CET, induced significant down-regulation of IE62 protein, which is essential for VZV lytic replication (Fig. 5).

\section{Effects of ester alkaloids from Cephalotaxus on replication of VZV clinical isolates}

To ascertain the effects of Cephalotaxus ester alkaloids on VZV-pOka-luciferase activity, qPCR was employed to assess activity against the clinical isolate of VZV (Fig. 6A). To this end, uninfected HFF cells were inoculated with VZV-YC01infected HFF cells at an MOI of 0.1, followed by treatment with DMSO, acyclovir, HT, HHT, or CET. Compared to $0 \mathrm{~h}$ after inoculation, the VZV DNA level was increased by 11,485fold in DMSO-treated cells at $72 \mathrm{~h}$ after inoculation. Similar to data obtained with VZV-pOka-luciferase, HT and HHT, but not CET, induced significant reduction of VZV-YC01 DNA by $57.8 \%$ and $97.1 \%$, respectively (Fig. $6 \mathrm{~A}$ ). The effects of HT and HHT on replication of VZV-YC01 were further determined using the plaque reduction assay (Fig. 6B). HFF cells were inoculated with serially diluted VZV-YC01-infected HFF cells, incubated for $6 \mathrm{~h}$ and treated with HT, HHT or CET at concentrations of $0,1,5,10$, or $25 \mathrm{ng} / \mathrm{ml}$. Cells were treated again with HT, HHT, or CET at $72 \mathrm{~h}$ after the initial treatment, and the number of plaques was counted to determine the titer expressed as plaque forming units $(\mathrm{pfu} / \mathrm{ml})$ at 6 days after infection via the plaque assay. Both HT and HHT treatment significantly reduced the number of plaques with an estimated $\mathrm{EC}_{50}$ of $16.15 \pm 1.94$ and $9.96 \pm$ $0.49 \mathrm{ng} / \mathrm{ml}$, respectively (Fig. 6B). The anti-VZV activities of HT and HHT were less effective against VZV-YC01 than VZV-pOka-luciferase. Nevertheless, both HT and HHT cle-
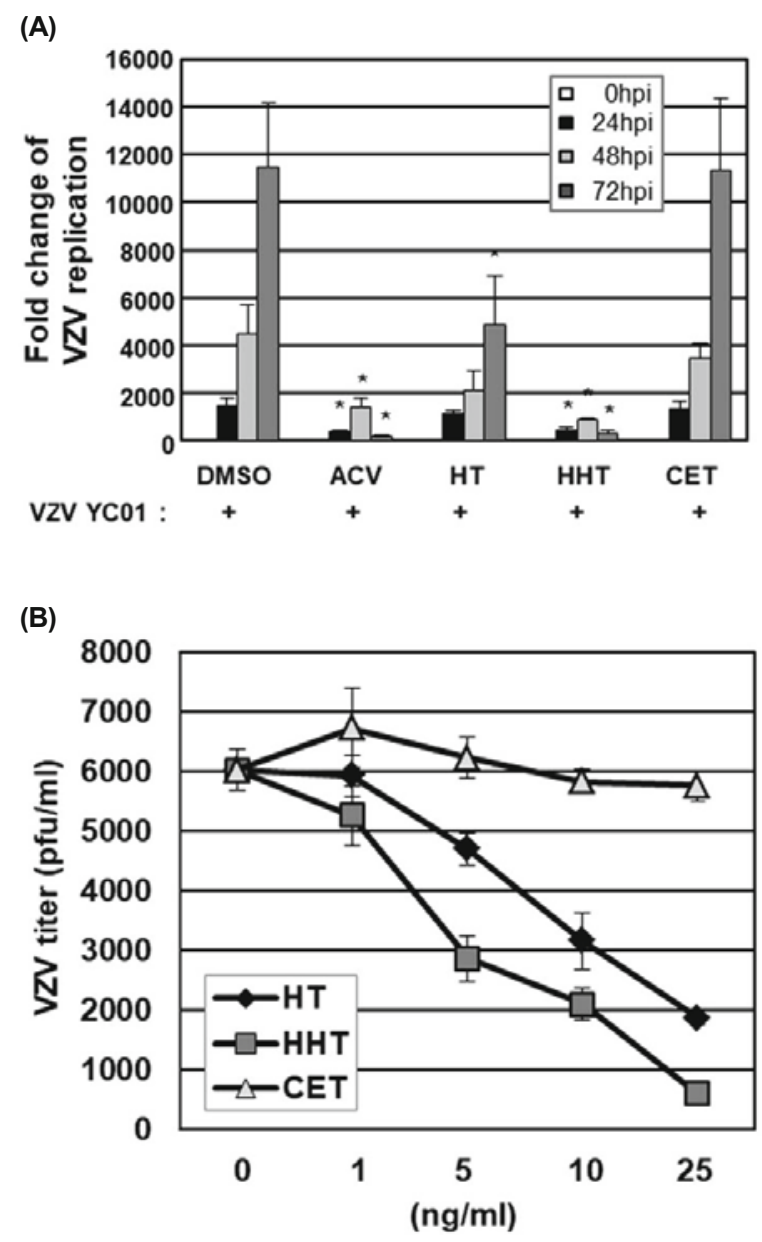

Fig. 6. Inhibition of VZV clinical isolate replication by HT and HHT. (A) HFF cells were inoculated with VZV-YC01-infected HFF cells at an MOI of 0.1 and treated with DMSO, HT, HHT, or CET (10 ng/ml). HFF cells treated with ACV $(5 \mu \mathrm{M})$ served as a positive control. At $72 \mathrm{~h}$ after inoculation, total DNA was harvested and the relative amounts of viral DNA measured using qPCR with primers specific for ORF62, as described in 'Materials and Methods'. The transcript levels at 0 hpi was set as 1 . (B) HFF cells were inoculated with serially diluted VZV-YC01-infected HFF cells, incubated for $6 \mathrm{~h}$ and treated with HT, HHT, or CET at concentrations of $0,1,5,10$, or $25 \mathrm{ng} / \mathrm{ml}$. Cells were treated again with HT, HHT or CET at $72 \mathrm{~h}$ after the initial treatment, and the number of plaques was counted to determine the titer expressed as $\mathrm{pfu} / \mathrm{ml}$ at 6 days after infection via the plaque assay. Data are represented as means \pm SD of three independent experiments. The asterisk $\left({ }^{*}\right)$ denotes a significant difference between samples determined using the two-sample $t$-test $(P<0.05)$. 
arly exerted strong inhibitory effects against both recombinant VZV-pOka-luciferase and the clinical isolate VZV-YC01.

\section{Discussion}

In the present study, we showed that the cephalotaxine esters, HT and HHT, inhibit replication of both the laboratory strain and clinical isolate of VZV in vitro. The finding that CET, the parental alkaloid for HT and HHT, exerted no antiVZV effects suggests that the ester side-chains at C-3 are critical for inhibition of VZV replication. ACV is widely used to treat $\mathrm{VZV}$-associated diseases, with a reported mean $\mathrm{EC}_{50}$ of $13 \pm 3 \mu \mathrm{M}$ for VZV-pOka (Morfin et al., 1999). Remarkably, HT and HHT were more potent than ACV in inhibiting VZV-pOka, with mean $\mathrm{EC}_{50}$ values of $18 \pm 6.5$ and $8.5 \pm 2 \mathrm{nM}$, respectively. Moreover, HHT was more potent than HT, indicating that, structurally, a methylene group in its side-chain may be responsible for enhanced anti-VZV activity.

Cephalotaxine esters are reported to possess antiviral as well as antileukemic activities (Romero et al., 2007; Kaur et al., 2013; Cao et al., 2015). Following screening of a natural product compound library, Kaur et al. (2013) demonstrated that HT inhibits replication of Chikungunya virus, a positivestrand RNA virus belonging to the Alphavirus genus of the Togaviridae family (Kaur et al., 2013). HHT has additionally been shown to exert antiviral effects against coronavirus, a positive-strand RNA virus, and HBV, a double-strand DNA virus (Romero et al., 2007; Cao et al., 2015). Another recent study demonstrated that HT inhibits replication of Sindbis virus, a member of the Togaviridae family (Jia et al., 2018).

Given that HT and HHT inhibit replication of both RNA and DNA viruses, antiviral activities are most likely to be attributable to effects on cellular rather than viral factor(s), which are critical for viral replication. Cephalotaxine esters have been shown to interfere with the elongation step of translation by blocking aminoacyl-tRNA binding to the acceptor site on the large ribosome subunit and peptide bond formation (Fresno et al., 1977). Kaur and co-workers suggested that HT suppresses Chikungunya virus replication by inhibiting the large ribosome subunit, in turn, down-regulating viral protein translation (Kaur et al., 2013).

Interestingly, HT and HHT significantly suppressed the mRNA levels of VZV lytic genes (Fig. 4). Thus, in addition to blocking ribosome function, these compounds may employ other mechanism(s) to inhibit VZV lytic gene expression and replication, which will be a focus of further studies. HT and HHT may interfere with signal transduction pathways and transcription factors involved in VZV lytic gene expression. Additionally, HT and HHT are reported to block cell cycle progression at $\mathrm{G}_{1} / \mathrm{S}$ or $\mathrm{G}_{2} / \mathrm{M}$ transition (Huang, 1975; Zhou et al., 1995). Since cell cycle regulation affects herpesvirus lytic gene expression and replication, one potential mechanism underlying inhibition of VZV replication by HT and HHT may be through effects on the cell cycle in infected cells.

\section{Acknowledgements}

This research was supported by the basic Science Research Program through the National Research Foundation of Korea (NRF) funded by the Ministry of Education, Science and Technology (NRF-2017R1D1A1B03032494). This work was also supported by the KIST Institutional Program (2E2783218-106). Jung-Eun Kim was supported by the National Junior Research Fellowship through the NRF funded by the Ministry of Education in Korea (2014H1A8A1021537).

\section{References}

Arvin, A.M. 1996. Varicella-zoster virus. Clin. Microbiol. Rev. 9, 361-381.

Arvin, A.M. 2002. Antiviral therapy for varicella and herpes zoster. Semin. Pediatr. Infect. Dis. 13, 12-21.

Arvin, A. and Gilden, D. 2013. Varicella-zoster virus, pp. 2015-2057. In Knipe, D.M. and Howley, P.M. (eds.), Fields virology, Philadelphia (Pennsylvania): Lippincott Williams \& Wilkins.

Bae, S., Kim, S.Y., Do, M.H., Lee, C.H., and Song, Y.J. 2017. 1,2,3,4,6penta-O-galloyl- $\beta$-D-glucose, a bioactive compound in Elaeocarpus sylvestris extract, inhibits varicella-zoster virus replication. Antiviral Res. 144, 266-272.

Bae, S. and Song, Y.J. 2017. Inhibition of varicellazoster virus replication by an ethanol extract of Lysimachia mauritiana. Mol. Med. Rep. 15, 3847-3851.

Cao, J., Forrest, J.C., and Zhang, X. 2015. A screen of the NIH clinical collection small molecule library identifies potential anticoronavirus drugs. Antiviral Res. 114, 1-10.

Chen, R., Guo, L., Chen, Y., Jiang, Y., Wierda, W.G., and Plunkett, W. 2011. Homoharringtonine reduced Mcl-1 expression and induced apoptosis in chronic lymphocytic leukemia. Blood 117, 156-164.

Cohen, J.I., Brunell, P.A., Straus, S.E., and Krause, P.R. 1999. Recent advances in varicella-zoster virus infection. Ann. Intern. Med. 130, 922-932.

Fresno, M., Jimenez, A., and Vazquez, D. 1977. Inhibition of translation in eukaryotic systems by harringtonine. Eur. J. Biochem. 72, 323-330.

Gnann Jr, J.W. 2007. Antiviral therapy of varicella-zoster virus infections. In Arvin, A., Campadelli-Fiume, G., Mocarski, E., Moore, P.S., Roizman, B., Whitley, R., and Yamanishi, K. (eds.), Human herpesviruses: Biology, therapy, and immunoprophylaxis, Cambridge, UK.

Huang, M.T. 1975. Harringtonine, an inhibitor of initiation of protein biosynthesis. Mol. Pharmacol. 11, 511-519.

Ida, M., Kageyama, S., Sato, H., Kamiyama, T., Yamamura, J., Kurokawa, M., Morohashi, M., and Shiraki, K. 1999. Emergence of resistance to acyclovir and penciclovir in varicella-zoster virus and genetic analysis of acyclovir-resistant variants. Antiviral Res. 40, 155-166.

Jeon, J.S., Won, Y.H., Kim, I.K., Ahn, J.H., Shin, O.S., Kim, J.H., and Lee, C.H. 2016. Analysis of single nucleotide polymorphism among varicella-zoster virus and identification of vaccine-specific sites. Virology 496, 277-286.

Jia, K., Yuan, Y., Liu, W., Liu, L., Qin, Q., and Yi, M. 2018. Identification of inhibitory compounds against Singapore grouper iridovirus infection by cell viability-based screening assay and droplet digital PCR. Mar. Biotechnol. (NY) 20, 35-44.

Kaur, P., Thiruchelvan, M., Lee, R.C., Chen, H., Chen, K.C., Ng, M.L., and Chu, J.J. 2013. Inhibition of chikungunya virus replication by harringtonine, a novel antiviral that suppresses viral protein expression. Antimicrob. Agents Chemother. 57, 155-167. 
Kim, J.E., Kim, S.Y., Lim, S.Y., Kieff, E., and Song, Y.J. 2014. Role of $\mathrm{Ca}^{2+}$ /calmodulin-dependent kinase II-IRAK1 interaction in LMP1-induced NF-kappaB activation. Mol. Cell. Biol. 34, 325334.

Lu, S. and Wang, J. 2014. Homoharringtonine and omacetaxine for myeloid hematological malignancies. J. Hematol. Oncol. 7, 2.

Morfin, F., Thouvenot, D., De Turenne-Tessier, M., Lina, B., Aymard, M., and Ooka, T. 1999. Phenotypic and genetic characterization of thymidine kinase from clinical strains of varicellazoster virus resistant to acyclovir. Antimicrob. Agents Chemother. 43, 2412-2416.

Powell, R.G., Weisleder, D., and Smith Jr, C.R. 1972. Antitumor alkaloids for Cephalataxus harringtonia: Structure and activity. J. Pharm. Sci. 61, 1227-1230.

Quintas-Cardama, A., Kantarjian, H., and Cortes, J. 2009. Homoharringtonine, omacetaxine mepesuccinate, and chronic myeloid leukemia circa 2009. Cancer 115, 5382-5393.
Romero, M.R., Serrano, M.A., Efferth, T., Alvarez, M., and Marin, J.J. 2007. Effect of cantharidin, cephalotaxine and homoharringtonine on "in vitro" models of hepatitis B virus (HBV) and bovine viral diarrhoea virus (BVDV) replication. Planta Med. 73, 552-558.

Tujebajeva, R.M., Graifer, D.M., Matasova, N.B., Fedorova, O.S., Odintsov, V.B., Ajtkhozhina, N.A., and Karpova, G.G. 1992. Selective inhibition of the polypeptide chain elongation in eukaryotic cells. Biochim. Biophys. Acta 1129, 177-182.

Zhang, Z., Rowe, J., Wang, W., Sommer, M., Arvin, A., Moffat, J., and Zhu, H. 2007. Genetic analysis of varicella-zoster virus ORF0 to ORF4 by use of a novel luciferase bacterial artificial chromosome system. J. Virol. 81, 9024-9033.

Zhou, D.C., Zittoun, R., and Marie, J.P. 1995. Homoharringtonine: An effective new natural product in cancer chemotherapy. Bull. Cancer 82, 987-995. 